ARTICLE

https://doi.org/10.1038/s41467-019-13893-w

\title{
Ultra-thin van der Waals crystals as semiconductor quantum wells
}

Johanna Zultak (10 1,2, Samuel J. Magorrian (10 1,2, Maciej Koperski (1) 1,2, Alistair Garner ${ }^{3}$, Matthew J. Hamer 1,2, Endre Tóvári'1,2, Kostya S. Novoselov (10 1,2, Alexander A. Zhukov1,2, Yichao Zou 2,3, Neil R. Wilson ${ }^{4}$,

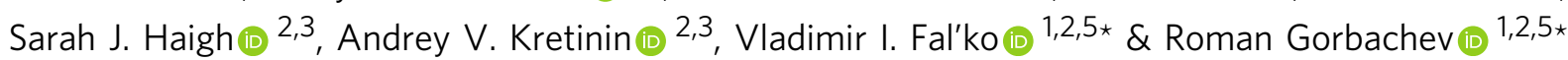

Control over the quantization of electrons in quantum wells is at the heart of the functioning of modern advanced electronics; high electron mobility transistors, semiconductor and Capasso terahertz lasers, and many others. However, this avenue has not been explored in the case of 2D materials. Here we apply this concept to van der Waals heterostructures using the thickness of exfoliated crystals to control the quantum well dimensions in few-layer semiconductor InSe. This approach realizes precise control over the energy of the subbands and their uniformity guarantees extremely high quality electronic transport in these systems. Using tunnelling and light emitting devices, we reveal the full subband structure by studying resonance features in the tunnelling current, photoabsorption and light emission spectra. In the future, these systems could enable development of elementary blocks for atomically thin infrared and $\mathrm{THz}$ light sources based on intersubband optical transitions in few-layer van der Waals materials.

\footnotetext{
${ }^{1}$ Department of Physics and Astronomy, University of Manchester, Oxford Road, Manchester M13 9PL, UK. ${ }^{2}$ National Graphene Institute, University of Manchester, Oxford Road, Manchester M13 9PL, UK. ${ }^{3}$ Department of Materials, University of Manchester, Oxford Road, Manchester M13 9PL, UK.

${ }^{4}$ Department of Physics, University of Warwick, Coventry CV4 7AL, UK. ${ }^{5}$ Henry Royce Institute for Advanced Materials, University of Manchester, Oxford Road, Manchester M13 9PL, UK. *email: vladimir.falko@manchester.ac.uk; roman@manchester.ac.uk
} 
$\mathrm{V}$ an der Waals crystals provide a platform for layer-by-layer material design which has expanded into a large multidisciplinary field during the last decade ${ }^{1-8}$. A broad variety of electronic and optoelectronic applications have been described and proof-of-concept devices have been reported ${ }^{2,5}$. Along with high quantum yield ${ }^{3}$, compact design, and mechanical flexibility ${ }^{5}$, one of the attractive features of two dimensional semiconductors (2DS) is their band-gap tunability. Due to the change in quantum confinement with thickness, the primary optical transition can be tuned over a broad energy range $\left(>1 \mathrm{eV}^{4,6,8}\right)$, and further fine adjustment is available through changes in dielectric environment ${ }^{7}$ and transverse electric fields ${ }^{9}$.

While the majority of 2DS demonstrate primary optical transitions in visible and near-infrared (IR) ranges, their intersubband transitions hold a largely unexplored potential to expand their optical activity further into the IR and THz. Depending upon the choice of material, number of layers and doping (n- or p-type), these subband transitions densely populate a region from $\sim 0.8 \mathrm{eV}$ for bilayers to $0.05 \mathrm{eV}$ for crystal thicknesess of $\sim 10$ atomic layers and can be utilized for optoelectronic devices ${ }^{10,11}$. Nonetheless, experimental studies of higher energy subbands remained elusive until recently, when the first experimental observation of intersubband transitions in few-layer $\mathrm{WSe}_{2}$ was demonstrated ${ }^{12}$ using near-field $\mathrm{THz}$ absorption spectroscopy. While $\mathrm{THz}$ spectroscopy gives access to direct measurements of some intersubband transitions, it only works in a small energy window and requires specific doping of the materials studied. At the same time, angleresolved photoemission spectroscopy (ARPES) only shows filled (valence) subbands and has diminished energy resolution when applied to small (micrometer-size) crystals ${ }^{13}$.

Here, we present an experimental approach that enables a comprehensive experimental study of the full subband structure of atomically thin 2DS, namely resonant tunneling spectroscopy combined with photoluminescence excitation (PLE) measurements. When applied to InSe, this method enables us to map the subbands on both conduction and valence band sides of the spectrum in agreement with theoretical band structure modeling and available ARPES data, as well as to trace the analogy between exfoliated atomically thin crystals and quantum wells in conventional semiconductors ${ }^{14}$.

\section{Results and discussion}

Experimental devices. Indium selenide is a new material in the 2DS family. It has a layer-dependent bandgap spanning from $1.2 \mathrm{eV}$ for bulk to almost $3.0 \mathrm{eV}$ for a monolayer ${ }^{6,15,16}$ and features a crossover ${ }^{13,17,18}$ from weakly indirect (in mono-, bi-, and tri-layer) to direct character (in crystals thicker than 4 layers). Its high crystal quality has recently been demonstrated, resulting in exceptionally high electron mobility ${ }^{6}$ making it a promising avenue for developing atomically thin nanoelectronics ${ }^{19}$. For resonant tunneling measurements we build van der Waals heterostructures, where exfoliated few-layer $\gamma$-InSe is sandwiched between hexagonal boron nitride (hBN) crystals to provide tunneling barriers, and we employ graphene layers as source and drain contacts on the top and the bottom of the device as illustrated in Fig. 1a. The thickness of hBN was selected to be 4-3 layers to allow for large bias voltages without sample overheating. These structures have been produced using the dry-stacking method in an argon environment ${ }^{20}$ to provide atomically clean and sharp interfaces, and to avoid InSe degradation ${ }^{21,22}$. The assembled stacks were deposited on an oxidized silicon wafer and contacts were defined using electron beam lithography (see Supplementary Figs. 1 and 2).

An optical micrograph of a typical device consisting of three InSe regions with number of layers $N=2,4$, and 5 shown in Fig. 1b. Each of these terraces has separate graphene contacts allowing them to have bias applied individually and control the position of the chemical potentials $\mu_{1}$ and $\mu_{2}$ in the source and drain graphene layers, respectively. To understand vertical charge transport in this system, we have reconstructed its band diagram using a tight-binding-calculated InSe band-structure ${ }^{6,18}$ and the relative band alignment measured in our recent ARPES studies ${ }^{13}$ and presented in Supplementary Fig. 4 using InSe exfoliated from the same bulk ingot. Here, the graphene Dirac point was near the NL-InSe (N layer-InSe) conduction band edge. The resulting band diagram is shown in Fig. 2 for a 4L-LED device in an unbiased (a), weakly biased (b), strongly biased in forward (c), and strongly biased in reverse configuration (d) 4L-InSe LED device (for the full band structure of InSe, see Supplementary Fig. 8).

Resonant tunneling spectroscopy. All these anticipated vertical transport regimes can be recognized in the experimentally measured tunneling current derivative $\left(\mathrm{d} I / \mathrm{d} V_{\mathrm{b}}\right)$ curves shown in Fig. 2e, $\mathrm{f}$ (for $I(V)$ curves see Supplementary Fig. 3). As soon as a small bias $\sim 0.3 \mathrm{~V}$ is applied, $\mathrm{d} I / \mathrm{d} V_{\mathrm{b}}$ shows a sharp increase (for $4 \mathrm{~L}-\mathrm{InSe}$ (e) and 5L-InSe (f)), indicating that $\mu_{1}$ crosses the edge of the first InSe conduction subband, in agreement with the band diagram in Fig. 2b. Upon further increase in $V_{\mathrm{b}}, \mathrm{d} I / \mathrm{d} V_{\mathrm{b}}$ displays a series of steps. We attribute this behavior to the step-like increase in the density of states of InSe when $\mu_{1}$ crosses the conduction subbands $c_{\mathrm{i}}$. Eventually, $\mu_{2}$ of the drain graphene

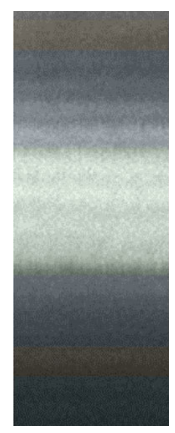

b

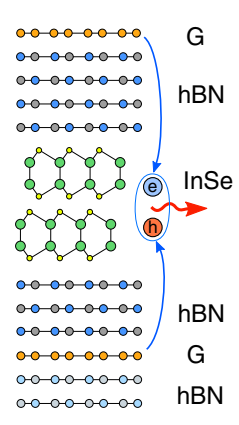

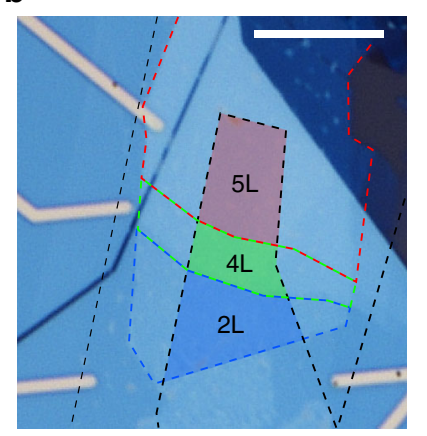

C

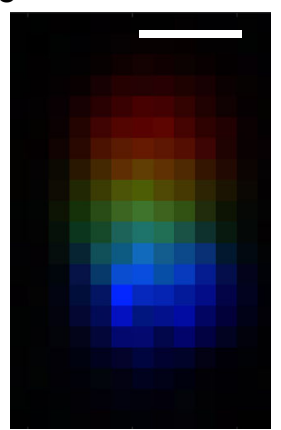

Fig. 1 InSe tunneling devices. a Cross-sectional annular dark-field scanning transmission electron image of a typical LED device with matching layer schematic on the right. b Optical micrograph of a device used to study resonant tunneling through InSe subbands. Graphene is outlined in black, 2 layer thick (2L) InSe in blue, $4 \mathrm{~L}$ in green and $5 \mathrm{~L}$ in red. c Artificially colored electroluminescence (EL) map of the device shown in b: blue corresponding to EL around $1.98 \mathrm{eV}$, green $1.50 \mathrm{eV}$ and red $1.35 \mathrm{eV}$ with sampling bandwidth of $1 \mathrm{meV}$ (detailed EL spectra can be found in SI). The scale bars are 10 m. 
a

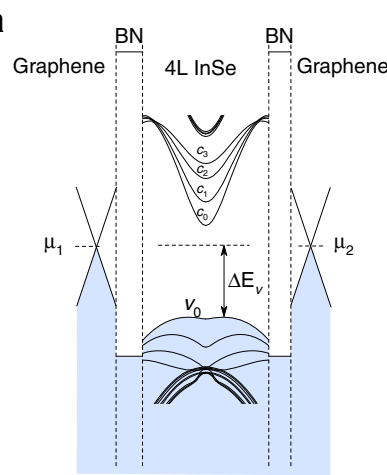

b

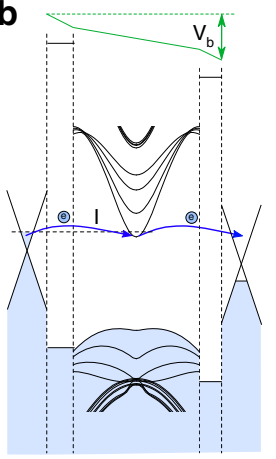

C

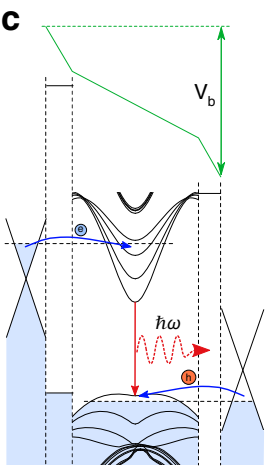

d

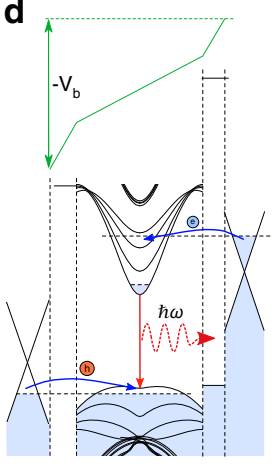

e

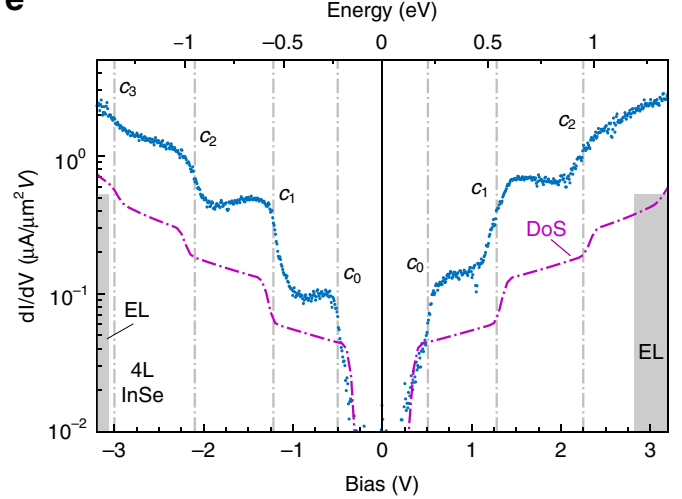

g

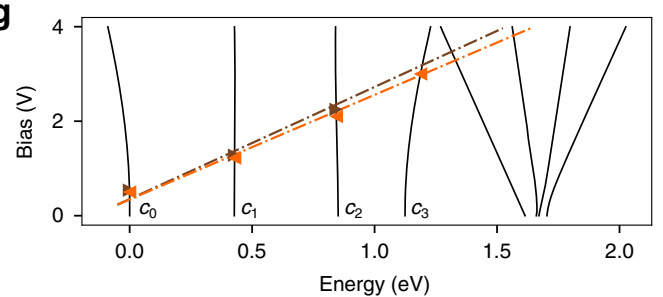

i

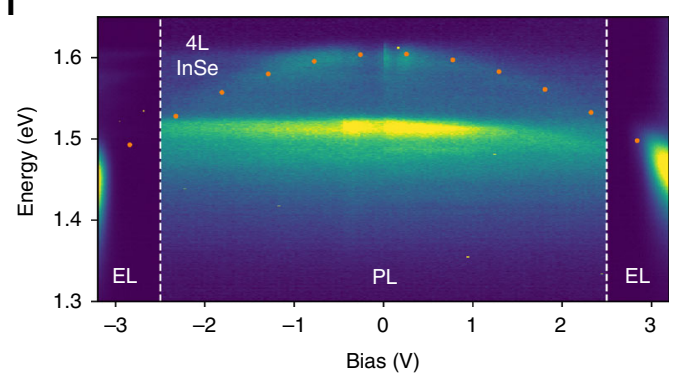

f

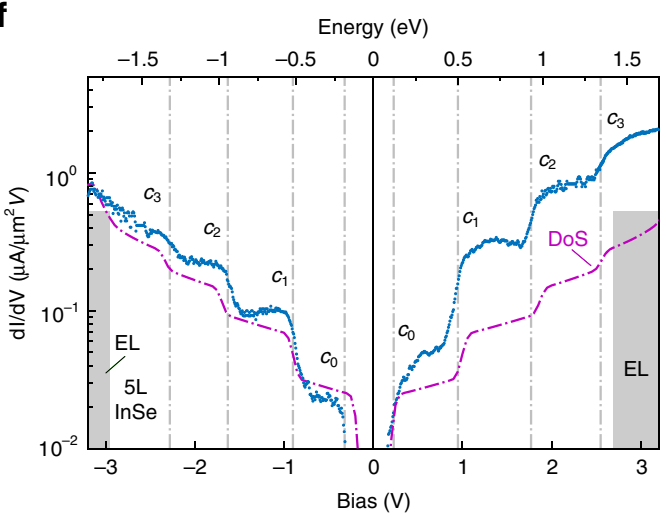

h

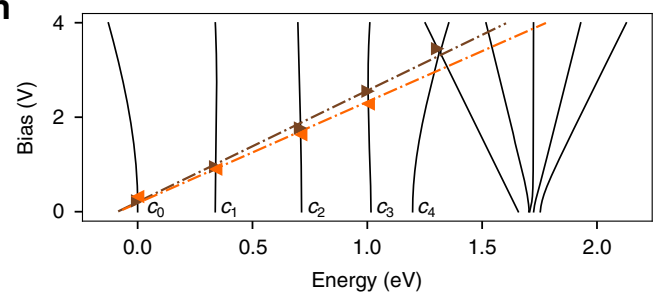

j

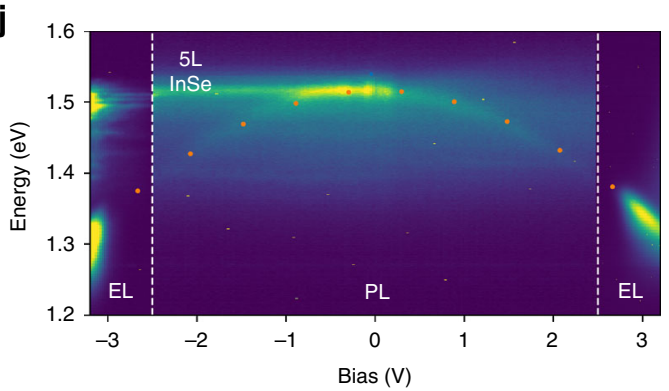

Fig. 2 Resonant tunneling spectroscopy of few-layer InSe crystals at $\mathbf{T}=\mathbf{4 . 2 K}$. Band alignment in $4 \mathrm{~L}$ InSe device, for unbiased (a), weakly biased (b) and strongly biased in forward (c) and reversed (d) configurations. $\mathrm{d} / / \mathrm{d} V_{\mathrm{b}}$ (blue) and density of states (DoS) of the conduction bands from tight binding model (purple, arb. u.) for $4 \mathrm{~L}$ (e) and $5 \mathrm{~L}$ (f) InSe film, with the number of layers established by AFM topography. Energy scale along the top axis was found using the EL onset values. Calculated evolution of the conduction subbands $c_{0, \ldots, N-1}$ with bias for $4 \mathrm{~L}(\mathbf{g})$ and $5 \mathrm{~L}$ (h) InSe (higher energy bands are also shown). Symbols indicate the bias at which the steps were measured in (e) and (f) for forward (brown) and reverse (orange) directions. Evolution of PL and EL with bias for $4 \mathrm{~L}(\mathbf{i})$ and $5 \mathrm{~L}(\mathbf{j})$ InSe. The orange dotted line indicates electrostatic calculations of the band gap reduction with electric field, non-dispersive line near $1.5 \mathrm{eV}$ is due to hydrocarbon contamination located outside the sample.

electrode reaches the top subband $\left(v_{0}\right)$ of the InSe valence band Fig. 2c, so that electrons and holes injected into the InSe from opposite sides can recombine, producing a bright electroluminescence (EL) signal, shown in the intensity map Fig. $1 c^{23}$. At this point the tunneling current density reaches $\sim 1 \mu \mathrm{A} \mu \mathrm{m}^{-2}$, and becomes unstable due to the contribution from the recombination process.
Using bias values at the onset of EL, $V_{\mathrm{b}}^{\text {th }}$, (indicated as gray shaded areas in Fig. 2e, f) we can determine the rate of change of the InSe potential with bias, as $\alpha=\Delta E_{v} / V_{\mathrm{b}}^{\text {th }}$, where $\Delta E_{v}$ is the offset between the Dirac point in graphene and the InSe valence band edge at zero bias ${ }^{13}$. The EL signal is observed to have a slight asymmetry for forward and reverse bias voltages due to the different hBN barrier thicknesses (4L and $3 \mathrm{~L}$ ) on either side: e.g. 
the thresholds of EL for $4 \mathrm{~L}$ InSe LED are $V_{\mathrm{b}}=2.83 \mathrm{~V}$ and $-3.02 \mathrm{~V}$. Therefore, the relation between $V_{\mathrm{b}}$ and energy in the band structure of the crystal is slightly different for forward and reverse bias (top axis in Fig. 2e, f). Using the experimentally determined values of the parameter $\alpha$ for both devices, we are able to compare the experimentally measured $\mathrm{d} I / \mathrm{d} V_{\mathrm{b}}$ with the theoretically calculated density of electron states (dashed purple line). We note that the linear density of states (DoS) in graphene makes the assignment of observed steps unambiguous, as its only feature, the Dirac point, is located near zero bias where the tunneling current is not measurable. This enables us to determine the energies of subband edges experimentally.

For completeness, in the theoretical analysis we took into account the shifts of both conduction and valence subbands caused by the out-of-plane electric field due to the applied bias as,

$$
E=\frac{V_{\mathrm{b}}}{d_{\text {InSe }}+\frac{\varepsilon_{\text {InSe }}}{\varepsilon_{\text {BN }}} d_{\mathrm{BN}}},
$$

where $d_{\text {InSe }}$ and $d_{\mathrm{BN}}$ are the respective total thicknesses of the InSe and $\mathrm{hBN}$ in the stack, and $\varepsilon_{\text {InSe }} / \varepsilon_{\mathrm{BN}} \approx 4.9$ is the ratio between the out-of-plane dielectric constants of InSe and hBN. This leads to a clearly pronounced quadratic red shift of the lowest subband's energy seen in Fig. $2 \mathrm{~g}$, h, similar to the previously observed quantum confined Stark effect in $\mathrm{MoS}_{2}$ monolayers $^{24}$. At the same time, subbands in the middle of the spectrum $\left(c_{1}, c_{2}\right.$ for $4 \mathrm{~L}$ and $c_{1}, c_{2}, c_{3}$ for $\left.5 \mathrm{~L}\right)$ remain almost constant, whereas the highest subband is blue shifted. The DoS calculations shown in Fig. $2 \mathrm{e}, \mathrm{f}$ take into account the bias dependence of the band structure and the resulting subband onsets match closely (within $40 \mathrm{meV}$ ) with the step-like $\mathrm{d} I / \mathrm{d} V_{\mathrm{b}}$ features observed in our experiment (shown as gray dashed lines at the highest slope).

The calculated subband shifts for both conduction and valence bands agree well with the shift in the photoluminescence (PL) energy shown in Fig. 2i, j. The observed evolution of EL with bias also accurately follows the trend predicted for PL and at $V_{\mathrm{b}} \approx 3 \mathrm{~V}$ is shifted by almost $200 \mathrm{meV}$ since the $c_{0}$ and $v_{0}$ bands move in opposite directions. It is also clearly non-symmetric, with an almost double EL linewidth for the negative biases, which can be explained with partial filling of the $c_{0}$ subband when the hBN barrier of the drain is thicker than that of the source. This also agrees with a much faster increase in EL intensity for negative $V_{\mathrm{b}}$, indicating that the recombination rate is primarily determined by a steeper rise in the DoS of the flatter valence band.

To illustrate the behavior of the subbands in the context of fewlayer InSe as a quantum well, we show in Fig. $3 \mathrm{~d}$ the $k_{z}$ dispersion of the bulk crystal together with the subband energies for 5-layer InSe at the in-plane $\Gamma$ point. The 5 -layer subband energies can be qualitatively matched with the bulk bands, quantized at 5 discrete $k_{z}$ momenta as is familiar in the analysis of subband spectra in semiconductor quantum wells ${ }^{25}$. We further illustrate the quantum well behavior of the subbands in Fig. $3 c$ by plotting conduction band wavefunction coefficients for $S$ orbitals on the (antisymmetric) indium pair in each layer, showing how the subband with the lowest effective $k_{z}$ has no nodes, with the number of nodes increasing with $k_{z}$.

Probing the valence subbands by PLE. Finally, we probed the subband states of InSe films using PLE, where the energy of the excitation light was continuously varied up to $2.7 \mathrm{eV}$ while
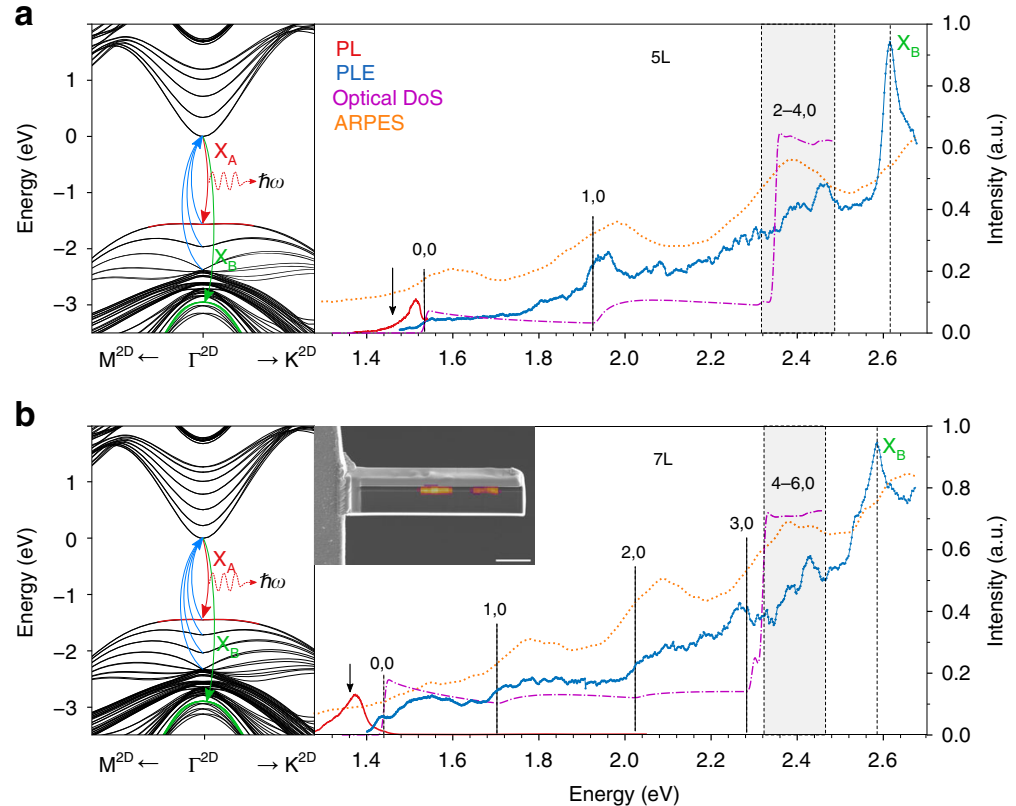
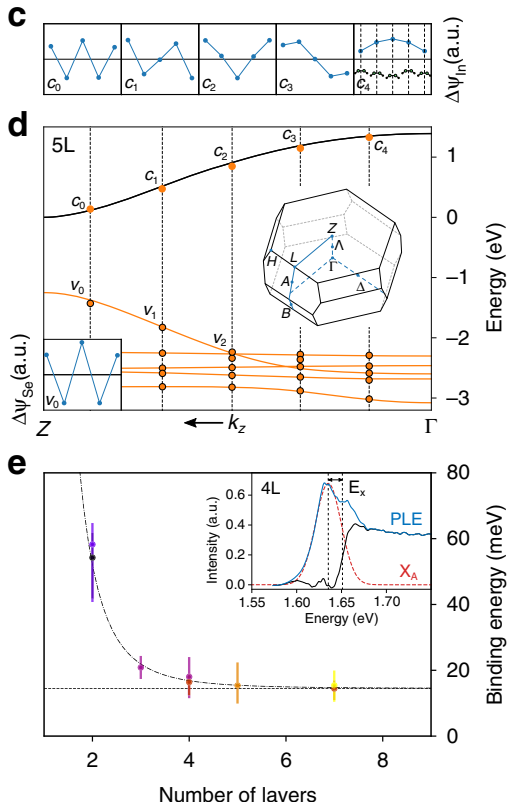

Fig. 3 PLE of 5L and $7 \mathbf{L}$ InSe at $\boldsymbol{T}=\mathbf{4 . 2 K}$. a, $\mathbf{b}$ Left $-2 \mathrm{D}$ band structure of $5 \mathrm{~L}$ and $7 \mathrm{~L}$ InSe around $\Gamma$ computed using DFT-parametrized tight-binding dispersion with spin-orbit interaction taken into account. Right - PL (red), PLE measured at the black arrow (blue) and projected optical DoS (purple) of lamellae for 5 and $7 \mathrm{~L}$ InSe. Relevant optical transitions from $v_{i}$ to $c_{j}$ are labeled as $i, j$. Orange dotted line shows ARPES intensity around $\Gamma$-point ${ }^{13}$, plotted to match $v_{0}-c_{0}$ optical transition in PLE with the first valence subband in ARPES. Inset shows scanning electron micrograph of the lamella overlaid with its PL map sampled at 1.30(2) eV, scale bar is $5 \mu \mathrm{m}$. c Amplitude $\Delta \psi_{\mathrm{In}}=\psi\left(\operatorname{In}_{1}\right)-\psi\left(\operatorname{In}_{2}\right)$, of $\ln S$-orbital contribution to the subbands $c_{n}$ across the layers for $N=5$. Band edges in bulk InSe are at Z points of the 3D Brillouin zone, hence the number of nodes in the layer-dependent microscopic wavefunction counts down from 4 for the lowest energy subband to 0 for the highest. d Solid lines - out-of-plane dispersion of bulk InSe at in-plane $\Gamma$ point. Orange dots $\Gamma$-point subbands for $5 \mathrm{~L} \ln S e$ at $k_{z}$ given by quantization of momentum in quantum well picture (dashed lines). Insets: center - 3D Brillouin zone of InSe, bottom left - amplitude $\Delta \psi_{\mathrm{Se}}=\psi\left(\mathrm{Se}_{1}\right)-\psi\left(\mathrm{Se}_{2}\right)$, of $\mathrm{Se} P_{z}$-orbital contribution to top valence subband. e Exciton binding energy ( $\left.E_{X}\right)$ extracted from PLE (see inset) as a function of number of layers in the film. The error bars correspond to the width of continuum absorption step. Colors indicate different samples. The dashed curve is displayed as a guide to the eye. 
the photoluminescence intensity of the lowest energy exciton $\mathrm{X}_{\mathrm{A}}$ was recorded. The resulting spectra shown in blue Fig. 3a, b for $N=5$ and 7 layers with the corresponding detection energy marked by the black arrow on the PL curve (red). Here, InSe films were encapsulated in thick $(>20 \mu \mathrm{m}) \mathrm{hBN}$ crystals without graphene contacts. The optical excitation from the lowest conduction band to the highest valence subband is mostly coupled to light polarized perpendicular to the plane of $2 \mathrm{D}$ crystal due to the intralayer electric dipole moment ${ }^{18}$. To provide both in- and outof-plane polarization of photons for the excitation, we have extracted a cross-sectional block (lamella), with an hBN/InSe/ $\mathrm{hBN}$ area of $2 \mu \mathrm{m} \times 20 \mu \mathrm{m}$ and a depth of $4 \mu \mathrm{m}$ using a focused ion beam instrument (see Supplementary Figs. 6 and 7) and rotated it $90^{\circ}$ such that the viewing direction is along the basal plane of the InSe crystal (for PLE spectra with in-plane polarized detection of $N=2$ to $N=7$ see Supplementary Fig. 5).

To interpret the PLE data we note that the material studied was lightly $\mathrm{p}$-doped, so that $\mathrm{X}_{\mathrm{A}}$ emission, used for detection involved equilibrium (rather than photoexcited) holes in the subband $v_{0}$ (dominated by the $\mathrm{P}_{z}$ orbitals of $\mathrm{Se}^{18}$ ). Due to the p-doping, $c_{0} \rightarrow v_{0}$ recombination is promoted, making PLE particularity sensitive to electron excitations $\left(v_{n} \rightarrow c_{0}\right)$ to the $c_{0}$ subband. As to $v_{n} \rightarrow c_{n}$ transitions, the photo-excited electrons have to undergo intersubband relaxation $\left(c_{n} \rightarrow c_{0}\right)$ before recombining radiatively with an equilibrium $v_{0}$ hole, suppressing their visibility in PLE due to the competition with non-radiative processes. The theoretically calculated projected optical DoS is plotted in Fig. 3a, $\mathrm{b}$ (purple dashed lines). It accounts for absorption of both outand in-plane polarized photons: including contributions from spin-flip interband transitions generated by spin-orbit mixing of $\mathrm{P}_{z}$ orbitals of Se with the $\mathrm{P}_{x, y}$ orbital (marked in green in Fig. 3a, inset). While such mixing is weak for the states in the highest subband $v_{0}$, it is enhanced for the deeper subbands, which are closer to the $\mathrm{P}_{x, y}$ band on the energy scale. The photon energy thresholds for each transition $v_{n} \rightarrow c_{0}$, determined from the spectra of standing waves subbands in the film, Fig. $3 c$, are indicated (as " $n, 0$ ") in Fig. 3a, b, and coincides with the features in the observed PLE spectra. For comparison, on the same plot we show micro-ARPES spectra measured on 5L and 7L films of InSe, which reflect the subband structure of the valence band in these films with noticeably broader features.

Also, on all samples studied, detailed inspection of the low energy onset of the PLE spectra have shown a noticeable excitonic peak followed by a plateau due to the free particle absorption. Measuring the difference between the peak energy (red line in the inset of Fig. 3e) and the onset of continuum absorption (black line $)^{26}$, we estimate the layer-dependent exciton binding energies as shown in Fig. 3e. The sharp increase of the binding energy for thin samples agrees with those typically seen in other $2 \mathrm{DS}^{27}$, while for thick flakes $(N>5)$ it closely matches (within $1 \mathrm{meV}$ ) that of bulk $\mathrm{InSe}^{28}$ indicated by dashed horizontal line in Fig. 3e.

\section{Discussion}

In summary, by the combined resonant tunneling and photoluminescence excitation spectroscopy approach we have achieved a comprehensive understanding of the entire subband spectrum of few-layer atomically thin films of InSe, including its evolution due to the quantum confined Stark effect. Having been exemplified here for InSe, this approach can be extended to a variety of $2 \mathrm{D}$ systems, including hetero- and homo-bilayers of transition metal dichalcogenides with a view to detect features of moiré superlattice minibands ${ }^{29}$. For optoelectronic applications, electrical injection of carriers in vertical devices is a possible route towards creating infrared and terahertz light sources that employ intersubband transitions. The above results demonstrate that, given appropriate doping to provide electrons or holes for recombination, one can control carrier injection into any designated subband of an atomically thin crystal to enable such emitters.

\section{Methods}

Device fabrication. For all devices Bridgman-grown bulk rhombohedral $\gamma$-InSe ${ }^{13}$ crystals were mechanically exfoliated onto PPC-coated (polypropylene carbonate) silicon wafers and crystals displaying large uniform terraces were identified using optical microscopy. These flakes were picked up with graphene/hBN stack carried on a PMMA membrane ${ }^{20}$ followed by a second thin hBN crystal and a graphene monolayer. Finally, the assembled stack was released onto a thick $(\sim 50 \mathrm{~nm}) \mathrm{hBN}$ exfoliated on an oxidized silicon wafer. The exfoliation and transfer of thin InSe took place in an argon filled glovebox to protect it from degradation ${ }^{19}$. Both graphene crystals were then contacted using electron beam lithography followed by $\mathrm{CHF}_{3}$ etching and $\mathrm{Cr} / \mathrm{Au}(1.5 \mathrm{~nm} / 50 \mathrm{~nm})$ deposition. Using an additional e-beam lithography step, the top graphene layer was divided into separate electrodes following outlines of the InSe terraces using $\mathrm{Ar} / \mathrm{O}_{2}$ plasma etching.

For the measurements of PL and PLE, devices without graphene have been made to prevent the emission quenching ${ }^{30,31}$. As before, InSe was encapsulated between hBN crystals and then covered with an additional thick hBN layer $(>200 \mathrm{~nm})$ followed by Cr/Au $(1 \mathrm{~nm} / 80 \mathrm{~nm})$ to prevent $\mathrm{Ga}^{+}$ion damage of the InSe during the milling process. Using FEI Helios dual-beam focused ion beam (FIB)/scanning electron microscope (SEM), an additional layer of platinum $(\sim 1 \mu \mathrm{m})$ was deposited over the selected region to provide further protection. Ga+ FIB milling was used to define the lamella at $30 \mathrm{kV}$, with current $7 \mathrm{nA}$ to remove bulk of the material and current $1 \mathrm{nA}$ to trim the lamella to its correct size. An OmniProbe micromanipulator was used to extract the lamella, rotate it by $90^{\circ}$, and position it on an OMICRON transmission electron microscopy (TEM) grid. After milling, the damaged edges of the $2 \mathrm{D}$ stack were removed by FIB polishing using decreasing acceleration voltages $(5 \mathrm{kV}, 47 \mathrm{pA}$ and $2 \mathrm{kV}, 24 \mathrm{pA})$. The final thickness of the specimen was $\sim 2 \mu \mathrm{m}$.

Optical measurements. All optical measurements have been performed at $4 \mathrm{~K}$ using an AttoDry100 cryostat and a AttoCFM I inset from Attocube. The illumination power on the sample was kept $\sim 0.4 \mathrm{~mW}$ and the spot size was $\sim 2 \mu \mathrm{m}$. For the excitation, a supercontinuum white light laser (Fianium WhiteLase WL-SC400-15-PP) combined with a Contrast filter (LLTF SR-VIS-HP8) have been used resulting in accurate wavelength control between $400 \mathrm{~nm}$ and $1000 \mathrm{~nm}$ with a linewidth of $\sim 2.5 \mathrm{~nm}$. For PLE measurements, the laser power was stabilized before the sample using an RD40-UV laser power controller from Brockton Electro-Optics Corp. The PL/PLE spectra were recorder using spectrometer diffraction grating (Princeton Instruments Acton Spectrapro SP-2500i with $300 \mathrm{~g} / \mathrm{mm}$ ) and nitrogen-cooled CCD camera (Princeton Instruments Pylon PIX-100BRX).

Tunneling spectroscopy. The tunneling measurements were carried out at $4.2 \mathrm{~K}$ by applying a DC bias and measuring the current between the source and drain graphene electrodes using a Keithley $2614 \mathrm{~B}$ source-meter.

Tight-binding model. Details concerning the spin-orbit coupling, the calculation of the density of states and the absorption spectra can be found in the Supplementary Fig. 9.

Cross-sectional STEM. For high resolution scanning transmission electron microscope (STEM) imaging the FIB cross section received further thinning with a Helios Dual beam FIB-SEM, using sequentially lower milling currents of $30 \mathrm{kV}, 16 \mathrm{kV}, 5 \mathrm{kV}$, and $2 \mathrm{kV}$ for ion beam milling and polishing. A probe side aberration corrected FEI Titan G2 80-200 kV was used with a probe convergence angle of $21 \mathrm{mrad}$, a HAADF inner angle of $48 \mathrm{mrad}$ and a probe current of $\approx 80 \mathrm{pA}$. To ensure the electron probe was parallel to the basal planes, the cross-sectional FIB sample was aligned to the relevant Kikuchi bands of the $\mathrm{Si}$ substrate and the $2 \mathrm{D}$ crystals.

\section{Data availability}

Additional data related to this paper may be requested from the authors.

Received: 19 July 2019; Accepted: 28 November 2019;

Published online: 08 January 2020

\section{References}

1. Geim, A. K. \& Grigorieva, I. V. Van der Waals heterostructures. Nature 499, 419-425 (2013).

2. Novoselov, K. S., Mishchenko, A., Carvalho, A. \& Castro Neto, A. H. 2D materials and van der Waals heterostructures. Science 353, aac9439 (2016). 
3. Britnell, L. et al. Strong light-matter interactions in heterostructures of atomically thin films. Science 340, 1311-1214 (2013).

4. Li, L. et al. Direct observation of the layer-dependent electronic structure in phosphorene. Nat. Nanotechnol. 12, 21-25 (2016).

5. Akinwande, D. et al. A review on mechanics and mechanical properties of $2 \mathrm{D}$ materials Graphene and beyond. Extreme Mech. Lett. 13, 42-77 (2017).

6. Bandurin, D. A. et al. High electron mobility, quantum Hall effect and anomalous optical response in atomically thin InSe. Nat. Nanotechnol. 12, 223-227 (2017).

7. Raja, A. et al. Coulomb engineering of the bandgap and excitons in twodimensional materials. Nat. Commun. 8, 15251 (2017).

8. Zhang, G. et al. Determination of layer-dependent exciton binding energies in few-layer black phosphorus. Sci. Adv. 4, eaap9977 (2018)

9. Haastrup, S., Latini, S., Bolotin, K. \& Thygesen, K. S. Stark shift and electricfield-induced dissociation of excitons in monolayer $\mathrm{MoS}_{2}$ and hBN/MoS Phys. Rev. B 94, 041401 (2016).

10. Magorrian, S. J., Ceferino, A., Zólyomi, V. \& Fal'ko, V. I. Hybrid $k \cdot p$ tightbinding model for intersubband optics in atomically thin InSe films. Phys. Rev. B 97, 165304 (2018).

11. Ruiz-Tijerina, D. A., Danovich, M., Yelgel, C., Zólyomi, V. \& Fal'ko, V. I. Hybrid $k \cdot p$ tight-binding model for subbands and infrared intersubband optics in few-layer films of transition-metal dichalcogenides: $\mathrm{MoS}_{2}, \mathrm{MoSe}_{2}$, $\mathrm{WS}_{2}$, and $\mathrm{WSe}_{2}$. Phys. Rev. B 98, 035411 (2018).

12. Schmidt, P. et al. Nano-imaging of intersubband transitions in van der Waals quantum wells. Nat. Nanotechnol. 13, 1035-1041 (2018).

13. Hamer, M. J. et al. Indirect to direct gap crossover in two-dimensional InSe revealed by angle-resolved photoemission spectroscopy. ACS Nano 13, 2136-2142 (2019).

14. von Klitzing, K. \& Mendez, E. Physics and Applications of Quantum Wells and Superlattices (Springer, 1987).

15. Mudd, G. W. et al. Tuning the bandgap of exfoliated InSe nanosheets by quantum confinement. Adv. Mater. 25, 5714-5718 (2013).

16. Lei, S. et al. Evolution of the electronic band structure and efficient photodetection in atomic layers of InSe. ACS Nano 8, 1263-1272 (2014).

17. Zólyomi, V., Drummond, N. D. \& Fal'ko, V. I. Electrons and phonons in single layers of hexagonal indium chalcogenides from ab initio calculations. Phys. Rev. B 89, 205416 (2014).

18. Magorrian, S. J., Zólyomi, V. \& Fal'ko, V. I. Electronic and optical properties of two-dimensional InSe from a DFT-parametrized tight-binding model. Phys. Rev. B 94, 245431 (2016).

19. Hamer, M. J. et al. Gate-defined quantum confinement in InSe-based van der Waals heterostructures. Nano Lett. 18, 3950-3955 (2018).

20. Frisenda, R. et al. Recent progress in the assembly of nanodevices and van der Waals heterostructures by deterministic placement of 2D materials. Chem. Soc. Rev. 47, 53-68 (2018).

21. Cao, Y. et al. Quality heterostructures from two-dimensional crystals unstable in air by their assembly in inert atmosphere. Nano Lett. 15, 4914-4921 (2015).

22. Rooney, A. P. et al. Observing imperfection in atomic interfaces for van der Waals heterostructures. Nano Lett. 17, 5222-5228 (2017).

23. Withers, F. et al. Light-emitting diodes by band-structure engineering in van der Waals heterostructures. Nat. Mater. 14, 301-306 (2015).

24. Roch, J. G. et al. Quantum-confined stark effect in a $\mathrm{MoS}_{2}$ monolayer van der Waals heterostructure. Nano Lett. 18, 1070-1074 (2018).

25. Lage, H., Heitmann, D., Cingolani, R., Grambow, P. \& Ploog, K. Center-ofmass quantization of excitons in GaAs quantum-well wires. Phys. Rev. B 44, 6550-6553 (1991).

26. Kittel, C. Introduction To Solid State Physics 8th edn (John Wiley \& Sons, 2003).

27. Wang, G. et al. Colloquium : excitons in atomically thin transition metal dichalcogenides. Rev. Mod. Phys. 90, 021001 (2018).

28. Camassel, J., Merle, P., Mathieu, H. \& Chevy, A. Excitonic absorption edge of indium selenide. Phys. Rev. B 17, 4718-4725 (1978).

29. Alexeev, E. M. et al. Resonantly hybridized excitons in moiré superlattices in van der Waals heterostructures. Nature 567, 81-86 (2019).

30. Gaudreau, L. et al. Universal distance-scaling of nonradiative energy transfer to graphene. Nano Lett. 13, 2030-2035 (2013).

31. Raja, A. et al. Energy transfer from quantum dots to graphene and $\mathrm{MoS}_{2}$ : the role of absorption and screening in two-dimensional materials. Nano Lett. 16, $2328-2333$ (2016).

\section{Acknowledgements}

We thank Alberto Morpurgo and Laurence Eaves for discussions and David Sanderson for critical reading of the manuscript. We acknowledge support from EPSRC grants EP/ N509565/1, EP/P01139X/1, EP/N010345/1, EP/P01139X/1 and EP/L01548X/1 along with the CDT Graphene-NOWNANO, and the EPSRC Doctoral Prize Fellowship. In addition, we acknowledge support from the European Graphene Flagship Project (785219), European Quantum Technology Flagship (820378) 2D-SIPC, MCA Scheme (751883), ERC Synergy Grant Hetero2D, ERC Starter grant EvoluTEM (715502), the ARCHER National UK Supercomputer RAP Project e547, Royal Society, US Army Research Office (W911NF-16-1-0279) and Lloyd Register Foundation Nanotechnology grant. We acknowledge support from Dr Alexei Barinov at Spectromicroscopy beamline, ELETTRA synchrotron, with the ARPES data.

\section{Author contributions}

V.I.F. and R.G. conceived the study; J.Z. fabricated devices with help from M.H.; J.Z. and M.K. performed measurements with help of E.T. and A.K.; J.Z. analyzed experimental data; S.J.H. and Y.Z. performed electron imaging; S.M. and V.I.F. provided theory used for the interpretation of the experiments; A.G. and A.Z. performed lamella preparation; N.W. provided ARPES data for comparison; V.I.F., R.G. and J.Z. wrote the manuscript with the help of S.M., A.K. and K.S.N.

\section{Competing interests}

The authors declare no competing interests.

\section{Additional information}

Supplementary information is available for this paper at https://doi.org/10.1038/s41467019-13893-w.

Correspondence and requests for materials should be addressed to V.I.F'k. or R.G.

Peer review information Nature Communications thanks Milan Radovic and the other anonymous, reviewer(s) for their contribution to the peer review of this work.

Reprints and permission information is available at http://www.nature.com/reprints

Publisher's note Springer Nature remains neutral with regard to jurisdictional claims in published maps and institutional affiliations.

(i)

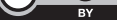
distribution and reproduction in any medium or format, as long as you give appropriate credit to the original author(s) and the source, provide a link to the Creative Commons license, and indicate if changes were made. The images or other third party material in this article are included in the article's Creative Commons license, unless indicated otherwise in a credit line to the material. If material is not included in the article's Creative Commons license and your intended use is not permitted by statutory regulation or exceeds the permitted use, you will need to obtain permission directly from the copyright holder. To view a copy of this license, visit http://creativecommons.org/licenses/by/4.0/.

(C) The Author(s) 2020 